\title{
Journalismus aus erzähltheoretischer Perspektive
}

Karl N. Renner, Johannes Gutenberg-Universität Mainz, Journalistisches Seminar

karl.n.renner@uni-mainz.de

\begin{abstract}
The essay demonstrates how the narratological theories developed in the field of literary studies can accomplish a better comprehension of journalistic narratives. As they have to accommodate the principles of journalism, these narratives have to be true, topical and impartial. From this point of view storytelling and the inverted-pyramid principle prove themselves as two complementary approaches of journalistic narration, whose stories are all constructed on the basis of real world occurrences and the ideologies of the diverse societies. Storytelling is enabling the communication of individual experience and the inverted-pyramid principle is supporting the narrative construction of future-open macro stories.
\end{abstract}

\section{Zusammenfassung}

Der Aufsatz zeigt am Beispiel der Zeitungsberichterstattung über die Guttenberg-Affäre 2011, wie die literaturwissenschaftliche Erzähltheorie zum besseren Verständnis des journalistischen Erzählens beitragen kann. Da dieses Erzählen den Anforderungen des Journalismus Rechnung tragen muss, müssen journalistische Erzählungen wahr, aktuell und unparteiisch sein. In diesem Zusammenhang erweisen sich Storytelling und Leadprinzip als zwei komplementäre Verfahren des journalistischen Erzählens, dessen Geschichten ausgehend von den Geschehnissen der realen Welt und den Weltbildern der jeweiligen Gesellschaften konstruiert werden. Dabei unterstützen das Storytelling die Vermittlung individueller Erfahrungen und das Leadprinzip die Erzählung zukunftsoffener Makrogeschichten.

\section{Keywords}

Erzähltheorie, Geschichte, Informationsjournalismus, Leadprinzip, Storytelling, Storyworld

\section{Journalismus und Erzählen}

Der Begriff «Geschichte» ist nach Beobachtung von Daniel Perrin die weitaus häufigste Ethnokategorie ${ }^{1}$, die Journalisten verwenden, wenn sie über ihre Arbeit sprechen (vgl. Perrin, 2010). Dennoch ist der Vorwurf, Journalisten würden keine Geschichten erzählen, sondern nur «anämisch» die Fakten berichten, ein durchgängiger Topos aller Streitschriften, die das journalistische Storytelling propagieren oder einen neuen, literarischen Journalismus fordern (vgl. Meier, 2004; Eberwein, 2013). So widersprüchlich beides ist,

1 Unter Ethnokategorien von Textformaten sind jene Kategorien zu verstehen, mit denen die Mitglieder einer Kommunikationsgemeinschaft selbst ihre Formate bezeichnen (vgl. Perrin 2010, S. 147). es zeigt, dass es für eine Betrachtung des Journalismus aus erzähltheoretischer Perspektive gute Gründe gibt. Denn der Begriff "Geschichte» ist ein zentraler Begriff der Erzähltheorie, wie das die Begriffsbestimmung belegt, die Silke Lahn und Jan Christoph Meister ihrer Einführung in die Erzähltextanalyse voranstellen: «Unter Erzählen verstehen wir das (sprachliche) Ausdrücken, Verknüpfen und gleichzeitige thematische Ordnen von (wahren oder vorgestellten) Fakten zu Geschichten» (Lahn \& Meister, 2008, S. 4; Hervorhebungen im Original).

Auch die historische Entwicklung des Journalismus legt es nahe, die journalistische Berichterstattung als eine besondere Form des Erzählens zu verstehen. Denn bis ins 18. Jahrhundert übernimmt der Journalismus seine Textmuster «von exis- 
tierenden literarischen Gattungen (...), z.B. von Erzählungen und Briefen» und viele Nachrichtenbeiträge «erinner[n] eher an eine Kleist'sche Novelle» als an heutige Nachrichtentexte (Burger, 1990, S. 8). Gerade die Gestaltung von Nachrichtentexten hat sich durch das Lead-Prinzip, wonach das Wesentliche zuerst kommt (vgl. Lüger, 1995, S. 94-102), so stark verändert, dass sie heute nicht mehr als Erzählungen gelten, auch wenn man auf den bunten Themenseiten viele "Soft News" findet, die nach wie vor als kurze Erzählungen gestaltet sind (vgl. Lüger, 1995, S. 103-108). Dieses Lead-Prinzip, das für die "Hard News" der Politik- und Wirtschaftsberichterstattung charakteristisch ist, wurde vom amerikanischen Journalismus während dessen Professionalisierungsschub in den 1880er Jahren entwickelt (vgl. Pöttker, 2003, S. 425). Wie denn generell anzunehmen ist, dass die Entwicklung und Fortentwicklung journalistischer Normen (vgl. Wilke, 2016) einen nachhaltigen Einfluss auf die Gestaltung der verschiedenen journalistischen Textsorten hatte.

Ohne diesen historischen Ansatz weiter zu verfolgen, prägender Faktor des journalistischen Erzählens ist zweifelsohne, dass dieses Erzählen den Anforderungen der Kommunikationsgattung Journalismus genügen muss. "Journalismus», so die Definition von Klaus Meier, ...

... recherchiert, selektiert und präsentiert Themen, die neu, faktisch und relevant sind. Er stellt Öffentlichkeit her, indem er die Gesellschaft beobachtet, diese Beobachtung über periodische Medien einem Massenpublikum zur Verfügung stellt und dadurch eine gemeinsame Wirklichkeit konstruiert. Diese konstruierte Wirklichkeit bietet Orientierung in einer komplexen Welt. (Meier, 2007, S. 13)

Versteht man daher Erzählen im Sinne von Lahn und Meister als eine kommunikative Handlung, die gleichermassen der Verknüpfung von Fakten zu Geschichten wie der Darstellung dieser Geschichten dient, dann ergibt sich die Frage, welche Auswirkungen es für die Konstruktion und die Präsentation von Geschichten hat, wenn dieses Erzählen den journalistischen Prin- zipien der Aktualität, derWahrheit und der Unparteilichkeit ${ }^{2}$ verpflichtet ist.

Als Grundlage zur Beantwortung dieser Frage soll die literaturwissenschaftliche Erzähltheorie dienen. Die Literaturwissenschaft beschäftigt sich immer schon mit dem Erzählen, machen doch Erzählungen der unterschiedlichsten Formen einen Grossteil ihres Fachgebietes aus. Dementsprechend elaboriert sind ihre erzähltheoretischen Konzepte (Hühn, Pier, Schmid, \& Schönert, 2009; Martínez, 2011). Ihre Anwendung auf das journalistische Erzählen, das ja kein fiktionales, sondern ein faktuales Erzählen ist und das nicht nur mit schriftlichen, sondern auch mit auditiven, visuellen und audiovisuellen Medien operiert, wird dadurch erleichtert, dass inzwischen interdisziplinäre Bestandsaufnahmen (Aumüller, 2012; Martínez, 2017) und Weiterentwicklungen vorliegen, die über das sprachliche und schriftliche Erzählen in literarischen Texten hinausführen. Für das journalistische Erzählen sind hier jene Arbeiten besonders wichtig, die das faktuale Erzählen (Klein \& Martínez, 2009; Fludernik \& Ryan, 2018) und das Erzählen mit Bildern und anderen Medien behandeln (Ryan, 2004; Kuhn, 2011).

Charakteristisch für die literaturwissenschaftliche Erzähltheorie ist zum einem die Auseinandersetzung mit dem Beziehungsfeld von Text, Autor, Erzähler und Leser und zum anderen die Differenzierung zwischen dem «Was der Erzählung», der histoire bzw. plot oder Geschichte, ${ }^{3}$ und ihrer sprachlichen Darstellung, dem «Wie der Erzählung», dem discours ${ }^{4}$ (vgl.

2 Das Prinzip der Unparteilichkeit wird in der Definition von Meier nicht explizit angeführt, es folgt jedoch aus dem Zusammenspiel des Relevanzkriteriums mit der Orientierungsfunktion des Journalismus.

3 Da sehr unterschiedliche Wissenschaftskulturen zur Erzähltheorie beigetragen haben, ist ihre Terminologie alles andere als einheitlich. Synopsen der wichtigsten Begriffe enthalten die Einführungsbände von Martínez \& Scheffel $(2012,28)$ und Lahn \& Meister (2008, S. 15-17).

4 Um Verwechslungen vorzubeugen, verwende ich die Schreibweise "discours" für den erzähltheoretischen und «Diskurs» für den 
Lahn \& Meister, 2008, S. 13-17; Martínez \& Scheffel, 2012, S. 22-28). Auf die Definition von Lahn und Meister bezogen, entspricht das "Wie der Erzählung» dem sprachlichen Ausdrücken von Geschichten und das «Was der Erzählung» dem Verknüpfen und Ordnen der Fakten zu einer Geschichte. Dabei konzentriert sich die strukturalistische Erzähltheorie auf die Organisation der Geschichte, während die neueren Ansätze die Gestaltung des discours und das Verhältnis von Autor, Text und Leser in den Vordergrund stellen. ${ }^{5}$

Die Unterscheidung dieser beiden Basiskategorien, des «Wie» und des «Was des Erzählens», erlaubt es, den Begriff des «journalistischen Erzählens» genauer zu fassen, womit sich auch die immer wieder aufflammenden Kontroversen zwischen den Anhängern der konventionellen Berichterstattung und des journalistischen Storytellings versachlichen lassen. Denn die beiden kommunikativen Handlungen «Berichten» und «Erzählen» sind zwei eng verwandte Assertionshandlungen, deren «Wie» sich zwar voneinander unterscheidet, doch deren «Was» in beiden Fällen eine Geschichte ist. Der Darstellungsmodus von Reportagen ist szenisch und schildernd (vgl. Lüger, 1995, S. 115), der von Nachrichten und Berichten dagegen sachlich und informierend (vgl. Lüger, 1995, S. 99). ${ }^{6}$ So gesehen lassen sich Reportagen als Erzählungen in einem engen Sinn und Nachrichten und Berichte als Erzählungen in einem weiten Sinn verstehen (vgl. Renner, 2012, S. 96-98). Ebenso wird nun verständlich, warum man immer wieder Beiträgen begegnet, die als Beispiele für journalistisches Storytelling gelten, obwohl sie keine Geschichten wiedergeben. Ihre Einordnung als Erzählung stützt sich nicht auf die Assertion einer Geschichte, soziolinguistischen Begriff.

5 Einen Überblick über die Geschichte der Erzähltheorie bieten Lahn \& Meister 2008, 19-34, eine Übersicht über die Hauptströmungen der modernen Erzähltheorie enthält Martínez 2011, S. 106-129.

6 Die Erzähltheorie diskutiert vergleichbare Unterschiede des Darstellungsmodus unter dem Begriffspaar "telling vs. showing" (vgl. Lahn \& Meister 2008, S. 26; Martínez \& Scheffel 2012, 50). sondern auf ihren szenischen Darstellungsmodus. ${ }^{7}$

Aus der Unterscheidung dieser beiden Basiskategorien folgt aber ebenso, dass sich eine Auseinandersetzung mit dem journalistischen Erzählen nicht auf die Fragen des Storytellings beschränken kann. Denn Erzählen ist nicht nur ein Akt der Präsentation, es ist immer auch ein Akt der Konstruktion von Geschichten. Im Folgenden soll am Beispiel der Berichterstattung über ein zentrales Ereignis der Plagiatsaffäre Guttenberg, der «Kelkheim-Episode», gezeigt werden, welche Folgen es für das Erzählen von Geschichten hat, wenn diese so konstruiert und dargestellt werden müssen, dass dies den journalistischen Kriterien der Wahrheit, der Aktualität und der Unparteilichkeit genügt. Das wird bei der Skandalberichterstattung besonders deutlich, wobei es von Vorteil ist, dass wir bei diesem Skandal den Standpunkt eines externen Beobachters einnehmen können. Denn anders als die Autoren der ausgewählten Artikel wissen wir heute, welches Ende der Skandal genommen hat.

\section{Die Kelkheim-Episode der Plagiatsaffäre Guttenberg}

Der Auftritt Guttenbergs am Montag, dem 21. Februar 2011, bei einer CDU-Veranstaltung in der hessischen Kleinstadt Kelkheim markiert aus heutiger Sicht den Wendepunkt der Plagiatsaffäre. Fünf Tage zuvor, am Mittwoch, dem 16. Februar 2011, hatte die Süddeutsche Zeitung erstmals über Unregelmässigkeiten in Guttenbergs Dissertation berichtet, was er - gerade auf dem Weg zu einem Truppenbesuch in Afghanistan - als «abstrus» zurückwies. Wieder zurück, kündigte er am Freitag an, dass er seinen Doktortitel bis zur Klärung

7 Ein Beispiel aus dem Materialkorpus dieser Studie ist die Reportage «Prüfungsdruck» von Tanjev Schultz (SZ 23.2.2011). Auch die Differenzierung von Narration und Storytelling, die Werner Früh vorschlägt, beruht darauf, dass der Begriff «Erzählen» sowohl auf inhaltliche wie auf Darstellungsaspekte bezogen werden kann (vgl. Früh \& Frey 2014, S. 86-91). 
der Vorwürfe ruhen lassen wolle. Nach einem oberflächlich ruhigen Wochenende sprach ihm an diesem Montag Bundeskanzlerin Merkel ihr Vertrauen aus. Sie habe ihn als Verteidigungsminister und nicht «als wissenschaftlichen Assistenten» berufen und das mache er gut. Am Abend erklärte dann Guttenberg in Kelkheim, wo er als Festredner eingeladen war, dass er in seiner Dissertation Fehler gemacht habe, dass er zu seinen Fehlern stehe und er daher für immer auf den Doktortitel verzichten wolle. Angesichts des jubelnden Beifalls seiner Anhänger, die sich dort um ihn geschart hatten, und der demonstrativen Unterstützung durch die Parteiführung schien die Affäre ausgestanden.

Ein objektiver Indikator für den besonderen Stellenwert der Kelkheim-Episode in der Plagiatsaffäre ist ihre Erwähnung in den Chroniken, die nach dem Ende dieses Skandals in verschiedenen Medien erschienen sind. Insgesamt wurden 13 Chroniken ausgewertet, zwölf davon erwähnen die Vorgänge in Kelkheim. Nur der Rücktritt Guttenbergs erreicht einen höheren Wert (vgl. Renner, 2013, S. 274).

Dieser Rücktritt erfolgte acht Tage später, am 1. März 2011. Trotz - oder wegen? - Guttenbergs brillanten Auftritts in Kelkheim nahm der öffentliche Druck auf ihn weiter zu. Die Enthüllungen der Online-Plattform Guttenplag über die Ungereimtheiten seiner Dissertation gingen unvermindert weiter, und nach der Äusserung der Kanzlerin flammten an immer mehr Universitäten Proteste auf. So sah sich Guttenberg schliesslich doch gezwungen, trotz Verzicht auf seinen Doktortitel als Verteidigungsminister zurückzutreten.

Als empirische Grundlagen dieser Studie dienen die Berichte, Reportagen und Kommentare in der Frankfurter Allgemeinen Zeitung und der Süddeutschen Zeitung über den Auftritt Guttenbergs in Kelkheim (vgl. die Liste im Anhang). Da diese Studie einen explorativen Anspruch hat, erscheint die geringe Zahl von Beispieltexten vertretbar. Auch wäre ein umfänglicheres Material im Rahmen dieser Untersuchung kaum zu bearbeiten, da sie erzähltheoretisch und nicht kommunikationswissenschaftlich-inhaltsanalytisch angelegt ist. Thematisch schliesst sie an meinen Aufsatz zur öffentlichen Konstruktion einer nicht-fiktionalen Geschichte an, der die Plagiatsaffäre insgesamt behandelt (Renner, 2013).

\section{Das "Was» des journalistischen Erzählens}

\subsection{Storyworld und Geschichte}

Hatte die Erzähltheorie das «Was der Erzählung» zunächst mit der erzählten $\mathrm{Ge}$ schichte identifiziert, so unterscheiden die neueren Arbeiten zwischen Geschichte und Diegese bzw. Storyworld, in der die Geschichte spielt: «Die Geschichte ist das Was der Erzählung - eine fiktionale Welt, die von Figuren und Dingen bevölkert ist und in der sich bestimmte Geschehnisse ereignen" (Lahn \& Meister, 2008, S. 14; Hervorhebungen im Original). Diese Begriffsdifferenzierung zeigt die Bedeutung, die das philosophische Konzept der möglichen Welten für das Verständnis des fiktionalen Erzählens gewonnen hat. Sie spiegelt aber auch wider, wie wichtig in unserer digitalen Medienkultur das transmediale Erzählen geworden ist, wobei es nicht mehr allein um das medienübergreifende Erzählen von Geschichten geht, sondern ebenso sehr um die Kreation von Figuren und um die Erfindung ganzer Welten (vgl. Thon, 2016).

Übernimmt man diese Differenzierung, kann man die Geschichte wiederum als eine Verknüpfung von Ereignissen in einer Storyworld definieren. Ereignisse werden dann «zur Einheit einer Geschichte integriert, wenn die Ereignisfolge zusätzlich zum chronologischen auch einen kausalen Zusammenhang aufweist, so dass die Ereignisse nicht nur aufeinander, sondern auch auseinander folgen» (Martínez \& Scheffel, 2012, S. 27).

Dieser kausale Zusammenhang wird von den verschiedenen erzähltheoretischen Konzepten mal aus einer internen, mal aus einer externen Perspektive erklärt.

Charakteristisch für die interne Perspektive ist, dass sie den inneren $\mathrm{Zu}$ sammenhang einer Geschichte auf die Intentionen der handelnden Figuren zu- 
rückführt. Das ist die Vorgehensweise der traditionellen Textinterpretation, aber auch innerhalb der strukturalistischen Erzählanalyse sind das Konzept der Handlungsalternativen von Claude Bremond (1972) und das Aktantenmodell von Algirdas Greimas (1972) diesem Ansatz verpflichtet. Konzepte, die eine externe Perspektive vertreten, erklären den kausalen Zusammenhang der Ereignisse mit der Relevanz, die die Abfolge der erzählten Geschehnisse «für die Bedeutungsabsicht des Erzähltextes» besitzt (Lahn \& Meister, 2008, S. 213). Es macht einen Unterschied, ob am Ende eines Krimis das Verbrechen aufgeklärt wird oder nicht. Für diese externe Perspektive stehen das Funktionenmodell von Vladimir Propp ([1928] 1975), die Mythenanalyse von Claude Lévi-Strauss ([1955] 1978), das Gleichgewichtskonzept von Tzvetan Todorov (1972) und der Sujetbegriff von Jurij M. Lotman (1972).

Will man diese literaturwissenschaftlichen Konzepte für das journalistische Erzählen fruchtbar machen, muss man die Storyworld journalistischer Erzählungen mit der empirisch vorgefundenen realen Welt identifizieren. Denn journalistische Erzählungen (im engen wie im weiten Sinne) sind faktuale Erzählungen. Aufgrund der elementaren Bedeutung, die das Wahrheitsprinzip für den Journalismus besitzt, haben sie den Anspruch, Geschehnisse und Geschichten zu erzählen, die tatsächlich stattgefunden haben oder stattfinden. Das unterscheidet sie kategorial von literarischen Erzählungen. Diese sind «fiktional in dem Sinne, dass sie grundsätzlich keinen Anspruch auf unmittelbare Referenzialisierbarkeit, d.h. Verwurzelung in einem empirisch-wirklichen Geschehen erheben» (Martínez \& Scheffel, 2012, S. 15).

Die Inhalte literarischer Erzählungen sind fiktiv, aber nicht fingiert in dem Sinne, dass sie ihre Leser täuschen wollen. Da sie keinen Wahrheitsanspruch haben, können sie auch nicht falsch sein und jemanden belügen (vgl. Martínez \& Scheffel, 2012, S. 15). ${ }^{8}$

8 Eine wesentliche Voraussetzung ist dabei, dass die Rezipienten den Unterschied zwischen faktualen und fiktionalen Erzählungen kennen. Exemplarisch demonstriert das
Identifiziert man die Storyworld von journalistischen Erzählungen mit der realen Welt, so folgt daraus, dass alle journalistischen Erzählungen das gleiche, gemeinsame Bezugsobjekt besitzen - es gibt nur eine reale Welt - und dass die Storyworld die unabhängige und die Erzählungen die abhängige Grösse sind. Bei fiktionalen Erzählungen entwickelt sich die Storyworld dagegen in Abhängigkeit von den jeweiligen Erzählungen. Daher kann jede Fortsetzung und jede mediale Bearbeitung eine etablierte Storyworld tangieren oder modifizieren. So entstehen bei Serien, Verfilmungen und anderen Formen des transtextuellen und transmedialen Erzählens immer wieder Mutationen und Verzweigungen der erzählten Storyworld (vgl. Ryan, 2013). Im Gegensatz dazu ist es für journalistische Erzählungen zwingend, dass sie auch dann zueinander passen, wenn sie von unterschiedlichen Autoren und in verschiedenen Medien erzählt werden. Abweichungen erfordern Revisionen oder gar die Preisgabe bereits erzählter Geschichten. ${ }^{9}$

Üblicherweise schreiben jedoch neue journalistische Erzählungen die alten einfach weiter fort. Das gilt insbesondere für die journalistischen Erzählungen im weiten Sinne. Nachrichten und Berichte sind in einem besonderen Masse dem Aktualitätsprinzip verpflichtet; da die Geschehensabläufe aber nur in Ausnahmefällen $\mathrm{zu}$ den Publikationsterminen passen, müssen Nachrichten und Berichte ihre Geschichten fast immer als Fortsetzungsgeschichten erzählen. Journalistische Erzählungen im engen Sinne können ihre Themen dagegen meistens so wählen,

Miguel de Cervantes in seinem Roman «Don Quijote».

9 Ein interessanter Grenzfall ist in diesem $\mathrm{Zu}$ sammenhang der Fernsehfilm «Der Minister», eine satirische Aufarbeitung der Plagiatsaffäre (Sat.1, 12. März 2013). Hier tragen zwar alle Figuren fiktive Namen, sind aber eindeutig mit realen Vorbildern identifizierbar. Andererseits erzählt der Film eine erfundene Geschichte: die "Ghostwriter-Story." Denn es wurde nie bewiesen, dass Guttenberg seine Dissertation von einem Ghostwriter schreiben liess, auch wenn dieser Verdacht immer wieder geäussert wurde. 
dass ihre Geschichten einigermassen abgeschlossen sind. Man muss daher beim journalistischen Erzählen Mikrogeschichten und Makrogeschichten unterscheiden, wenn eine Geschichte nicht von einem einzelnen Text, sondern im Rahmen des journalistischen Diskurses erzählt wird. «Einzelne journalistische Texte, ob narrativ oder nicht, können als Bestandteil eines narrativen Makrotextes, einer Makroerzählung aufgefasst werden, die ihrerseits durchaus einer narrativen Struktur (Komplikation/Auflösung) folgt» (Robert, 2013, S. 53).

\subsection{Zur Konstruktion journalistischer Geschichten: Geschichte und Welt}

Die Identifikation der Storyworld journalistischer Erzählungen mit der realen Welt macht es weiterhin erforderlich, strikt zwischen der Konstruktion von Geschichten auf Seiten der Rezipienten und der Produzenten zu unterscheiden. Die Rezipienten konstruieren die Geschichten, die ihnen erzählt werden, auf Grundlage kommunizierter Texte. Die Journalisten, die Produzenten dieser Texte, konstruieren ihre Geschichten jedoch aufgrund von Ereignissen in der realen Welt. Sei es, dass sie bei ihren Recherchen diese Ereignisse als Augenzeugen selbst beobachten, sei es, dass sie sich auf zuverlässige Quellen stützen. Daher kann man mit erzähltheoretischen Konzepten, die die Konstruktion der Geschichten ausgehend von der Textrezeption erklären (wie das für die meisten literaturwissenschaftlichen Ansätze zutrifft), zwar die Rezeption journalistischer Erzählungen, nicht aber ihre Produktion erfassen.

Hier bietet eine Verknüpfung von literaturwissenschaftlicher und kognitiver Erzähltheorie, wie das Marie-Laure Ryan skizziert, eine Lösung. Ryan unterscheidet zum einem zwischen den beiden Eigenschaften "being a narrative" und "possessing narrativity," und zum anderen sind Geschichten ihrer Auffassung nach "in two distinct realms" zu lokalisieren.

On one hand, narrative is a textual act of representation (...). On the other hand, narrative is a mental image - a cognitive construct - built by the interpreter as a response to the text. (...) But it does not take a representation proposed as a narrative to trigger the cognitive construct that constitutes narrativity: we may form narrative scripts in our mind as response to life, which is definitely not a representation. (Ryan, 2004, S. 9)

Folgt man diesem Vorschlag, so sind journalistische Geschichten zunächst Konstruktionen der Autoren, die diese Geschichten aufgrund ihrer Beobachtungen in der realen Welt erzählen. Diese Geschichten werden dann von den Rezipienten auf Grundlage der kommunizierten Texte rekonstruiert. Dabei ist es unerheblich, ob die Autoren ihre Geschichten ausgehend von den Beobachtungen bottom-up konstruieren, ob sie top-down ihren Beobachtungen ein Handlungsschema (vgl. Martínez \& Scheffel, 2012, S. 127) überstülpen oder ob es sich um wechselseitige Prozesse handelt. Entscheidend ist vielmehr, dass man demnach nicht nur die Geschichten fiktionaler, sondern auch die Geschichten faktualer Erzählungen als Konstruktionen betrachten muss, obwohl man diese intuitiv viel eher mit den stattgefundenen Geschehnissen identifizieren möchte.

Dass die Geschichten faktualer Erzählungen keine Widerspiegelungen der Wirklichkeit, sondern Konstrukte ihrer Erzähler sind, ist bei Erzählungen vor Gericht besonders auffällig. Kläger und $\mathrm{Be}$ klagte, Ankläger und Verteidiger fügen das umstrittene Geschehen ihren Standpunkten entsprechend $\mathrm{zu}$ Geschichten und Gegengeschichten zusammen und müssen dazu keineswegs Lüge und Wahrheit vermengen. Geschichten können auch dann falsch sein, wenn die Einzelheiten stimmen. Die Aufgabe des Richters ist es dann, aus diesen «konkurrierenden Narrativen» ein «Masternarrativ» $\mathrm{zu}$ formen, das für alle Beteiligten verbindlich ist und Grundlage des Urteils bildet (von Arnauld, 2009, S. 32).

In ähnlicher Weise benutzen auch bei Skandalen die gegnerischen Lager die gleichen Gegebenheiten, um Geschichten und Gegengeschichten zu konstruieren. Doch anders als beim Erzählen vor 
Gericht sind dies keine zurückliegenden und abgeschlossenen Geschichten. Diese Geschichten entwickeln sich mit dem Fortgang des Skandals, sie sind erst dann abgeschlossen, wenn der Skandal beendet ist. Während des Skandals sind sie zukunftsoffen und verbinden vergangene Ereignisse mit aktuellen Handlungsalternativen und zukünftigem Geschehen. Daher entstehen diese Geschichten nicht nur allein durch ihre Erzählung, sie entstehen ebenso durch die Handlungen der beteiligten Akteure, wobei der Sinn dieser Handlungen nur bedingt von den Intentionen der jeweiligen Akteure abhängig ist. Er ergibt sich viel eher durch den Stellenwert, den diese Handlungen für den Fortgang der jeweiligen Geschichte besitzen. In literarischen Erzählungen ist die narrative Sanktion ein vergleichbares Phänomen dieser Sinngebung aus externer Perspektive. Normverletzungen werden nicht mit den diskursiven Mitteln des Erzählers, sondern durch den weiteren Gang der Ereignisse verurteilt (vgl. Krah, 2006, S. 333).

«Skandalgeschichten» sind also für das Verständnis des journalistischen Erzählens in doppelter Hinsicht aufschlussreich. In erzähltheoretischer Hinsicht bieten sie eine Möglichkeit, die Konstruktion faktualer Geschichten transparent zu machen. In journalismustheoretischer Hinsicht machen sie das immanente Spannungsverhältnis zwischen den journalistischen Prinzipen der Wahrheit, Aktualität und Unparteilichkeit deutlich. Zugleich zeigen sie auf, wie der Journalismus dieses Spannungsverhältnis mithilfe der Trennung von Information und Meinung ${ }^{10}$ so auszutarieren sucht, dass er seiner gesellschaftlichen Funktion gerecht wird, durch die von ihm konstruierte Wirklichkeit in einer komplexen Welt Orientierung zu bieten (vgl. Meier, 2007, S. 13). In der Skandalberichterstattung ist das alles besonders ausgeprägt, es trifft aber mehr oder minder stark auch für viele andere

10 Die Trennungsnorm entwickelt sich genauso wie das Leadprinzip während des Professionalisierungsschubs des amerikanischen Journalismus um 1880 (vgl. Emery \& Emery 1992, S. 182).
Bereiche der journalistischen Berichterstattung zu.

\subsection{Geschichten und Gegengeschichten in der Plagiatsaffäre I}

Im Skandal, den Guttenbergs Dissertation auslöst, konkurrieren drei (Makro-) Geschichten miteinander: die Fehler-, die Betrugs- und die Hetzjagdgeschichte (vgl. Renner, 2013, S. 276-283). Die Fehlergeschichte erklärt die Ungereimtheiten der Dissertation als Fehler beim Schreiben der Doktorarbeit. Die Betrugsgeschichte geht davon aus, dass Guttenberg betrogen hat. Die Hetzjagdgeschichte begründet die Plagiatsvorwürfe gegen Guttenberg damit, dass es seinen Kritikern nicht um die Reinheit der Wissenschaft gehe, sondern «darum, einen Superstar zu entzaubern", so die Post von Wagner in der BILD-Zeitung vom 17. Februar 2011. Wie die Berichterstattung zeigt, setzt sich Guttenberg in seiner Kelkheimer Rede mit allen drei Geschichten auseinander und strickt sie weiter fort.

An erster Stelle ist die Fehlergeschichte zu nennen. Guttenberg bestätigt sie, indem er die Konsequenzen zieht, die sich für ihn aus dieser Geschichte ergeben: Er verzichtet in aller Öffentlichkeit auf seinen Doktortitel. Damit scheint die Plagiatsaffäre ihren Abschluss zu finden. Dementsprechend hervorgehoben ist die Fehlergeschichte in der Frankfurter Allgemeinen Zeitung und in der Süddeutschen Zeitung. Sie bestimmt die Überschriften der Artikel, mit denen beide Zeitungen über Guttenbergs Auftritt berichten: Guttenberg gibt «gravierende Fehler» zu (FAZ 22.2.2011), Guttenberg gibt seinen Doktortitel zurück (SZ 22.2.2011). Die Betrugsgeschichte, mit der Guttenbergs Gegner ihre Rücktrittsforderungen begründen, spielt in der Kelkheimer Rede wiederum deswegen eine Rolle, weil Guttenberg diese Geschichte entkräften muss. Er habe, so liest man in der FAZ, "die Fehler «nicht bewusst gemacht und nicht absichtlich getäuscht). (...) Von einem Rücktritt als Verteidigungsminister sprach er nicht" (FAZ 22.2.2011). Und die SZ schreibt, Guttenberg zufolge seien die falschen Zitate nicht bewusst geschehen. 
«Einen Rücktritt schloss Guttenberg aus» (SZ 22.2.2011). Die Reportage in der Süddeutschen Zeitung vom 23.2.2011 führt dann vor, wie die Hetzjagdgeschichte dazu dient, Guttenberg von den Betrugsvorwürfen zu entlasten. "Und die erdrückenden Beweise, die Doktorarbeit im Kopier-Verfahren verfasst zu haben?», fragt der Reporter. "Das ist nichts als politische Intrige. Da, wo Guttenberg ist, da wollen doch alle hin», lautet die Antwort des Befragten, der davon überzeugt ist, "so einer wie der (...), der kann auch Kanzler werden.» Auch Guttenberg selbst bedient diese Geschichte. Die FAZ zitiert: «Das Land müsse aufpassen, dass sich seine «Massstäbe nicht verschieben>, sagte er und kritisierte, dass die ‘Fussnoten in einer ministeriellen Doktorarbeit> zeitweilig mehr Aufmerksamkeit erhalten hätten als die getöteten deutschen Soldaten in Afghanistan» (FAZ 22.2.2011). Und die SZ berichtet: «Umjubelt von der CDU-Basis rief er: «Eine oberfränkische Wettertanne hauen solche Stürme nicht um.»" (SZ 22.2.2011).

Betrachtet man die Kelkheim-Berichterstattung aus heutiger Perspektive, so sind vor dem Hintergrund dieser drei konkurrierenden Geschichten deutlich die Probleme zu erkennen, mit denen die Journalisten in der damaligen Situation zurechtkommen müssen.

Journalisten sind dem Aktualitätsprinzip verpflichtet, daher sind viele Geschichten, die sie erzählen müssen, zukunftsoffen. Anders als Historiker kennen sie nicht das Ende, auch können sie bei konkurrierenden Geschichten nicht abwarten, welche Geschichte sich letztlich als die verbindliche erweist. Ebenso wenig können sie bei konkurrierenden Geschichten die Ereignisse nach dem Muster einer der Geschichten zu einem zusammenhängenden Ganzen verbinden, ohne damit für die Anhänger des entsprechenden Lagers Partei zu ergreifen. Dies widerspricht zwar nicht dem Selbstverständnis des Meinungsjournalismus, wie das in diesem Fall die Berichterstattung der BILD-Zeitung vorführt, doch mit der Objektivitätsnorm des Informationsjournalismus ist das unvereinbar. $^{11}$

Weiterhin ist zu sehen, wie auch die journalistische Wahrheitspflicht die Konstruktion journalistischer Geschichten tangieren kann. Denn die Journalisten können zum damaligen Zeitpunkt trotz all ihrer Recherchen nicht verbindlich die Frage beantworten, die im Mittelpunkt der Plagiatsaffäre steht: Hat Guttenberg betrogen oder nicht? Dazu fehlt ihnen wie bei jeder Verdachtsberichterstattung (vgl. Liesem, 2017) die erforderliche Legitimation. So können sie zwar über alle Sachverhalte berichten, die für einen Betrug sprechen, doch das Vorliegen eines Betrugs selbst können sie erst dann behaupten, wenn Guttenberg dies selbst einräumt oder wenn dieser Verdacht in einem offiziellen Verfahren bestätigt wurde. Ein solches Verfahren kann aber nur von einem Gericht oder von der Universität Bayreuth durchgeführt werden, die den Doktortitel verliehen hat. Bis dahin können Journalisten nur Vermutungen anstellen. Das sind aber keine Fakten, die sie für die Konstruktion einer Geschichte verwenden dürfen.

Die Berichterstattung über Kelkheim ist aber auch ein gutes Beispiel dafür, wie der (Informations-)Journalismus diese Probleme durch die Trennung von Information und Meinung zu lösen sucht. In ihren Informationsartikeln berichten die beiden Zeitungen über den Auftritt Guttenbergs und die weiteren Fakten, die zur Beurteilung dieses Ereignisses relevant sind. Sie zitieren die aktuellen Äusserungen führender Politiker, sie informieren über den aktuellen Stand der Textvergleiche bei Guttenplag und sie melden, dass die Universität Bayreuth Guttenbergs Bitte um die Aberkennung seines Doktortitels bestätigt hat. Dabei rekurrieren sie in der für Nachrichten typischen Weise immer wieder auf das Vorwissen ihrer Leser (vgl. Lüger, 1995, S. 99). Die Bewertung der berichteten Fakten erfolgt dann in den Meinungsartikeln. Exemplarisch ist der Kommentar von Berthold Kohler, den die FAZ am

$11 \mathrm{Zu}$ den verschiedenen Varianten des Journalismus vgl. Meier (2007, S. 78-79 und 183187). 
23.2.2011 unter dem Titel Entlassen veröffentlicht. Kohlers argumentative Basis ist die Betrugsgeschichte, auch wenn diese damals nicht als die allgemein verbindliche Geschichte akzeptiert ist. Er stellt die Selbstinszenierung Guttenbergs als «Saubermann» infrage und fordert den Rücktritt des Ministers: «Guttenberg hätte sich selbst, den Werten, die er hoch hält, und langfristig auch der Union einen besseren Dienst erwiesen, wenn er nicht nur der Universität Bayreuth einen Brief geschrieben hätte, sondern auch der Kanzlerin» (FAZ 23.2.2011).

Schliesslich zeigt die Kelkheim-Berichterstattung auch, warum es für den (Informations-)Journalismus so schwierig ist, Geschichten attraktiv und spannend zu erzählen. Das Trennungsgebot verhindert, dass die Akteure der jeweiligen Geschichten moralisch bewertet werden. Man kann daher nicht positive Helden und Bösewichter gegeneinanderstellen, ohne sich einem Manipulationsverdacht auszusetzen. Auch kann man den Handlungsbogen der erzählten Geschichten nur selten schliessen, da deren Ende aufgrund des Aktualitätsprinzips oftmals noch offen ist.

Bei Reportagen wird dieses Problem dadurch gelöst, dass anstelle der gesamten Geschichte eine in sich abgeschlossene Episode erzählt wird und dabei alles andere nur erwähnt wird. So verwenden sowohl Oliver Georgi wie auch Thorsten Schmitz und Stefan Braun die Dramaturgie des Kelkheimer Auftritts als Handlungsbogen der Geschichten, die sie in ihren Reportagen Die fränkische Wettertanne nach dem Sturm (FAZ 23.2.2011) und Der Doktor und das liebe Volk (SZ 23.2.2011) erzählen. Georgi beschränkt sich dabei auf das Geschehen in Kelkheim, den feierlichen Einzug in die Halle, die Rede und den jubelnden Schlussapplaus. Schmitz und Braun verbinden es mit der herzlichen Begrüssung, mit der die Abgeordneten der Unionsfraktion am nächsten Tag Guttenberg in Berlin empfangen. Tanjev Schultz kann dagegen in seiner Reportage Prüfungsdruck über die Situation an der Universität Bayreuth (SZ 23.2.2011) auf keinen linearen Geschehensablauf zurückgreifen, um einen Handlungsbogen $\mathrm{zu}$ bilden. So spitzt er seine Reportage auf eine Schlusspointe zu: An der juristischen Fakultät wurde vor kurzem ein Graduiertenkolleg für geistiges Eigentum eingerichtet. «Das Kolleg bildet Doktoranden aus - lauter Experten für geistiges Eigentum. Diethelm Klippel [der Leiter des Graduiertenkollegs, knr] muss selbst lachen, als er davon erzählt.»

\subsection{Zur Konstruktion journalistischer Geschichten: Erzähler und Geschichte}

Identifiziert man die Storyworld faktualer Erzählungen mit der realen Welt, so hat das weitreichende Auswirkungen für die Referenz dieser Erzählungen. Nicht weniger weitreichend sind die Folgen für die Beziehung zwischen Erzähler und Geschichte. Denn dann gehören die Erzähler mitsamt ihren Geschichten zur real existierenden Welt. Daher müssen sie, anders als Märchen- oder Romanerzähler, für die Wahrheit dieser Geschichten haften. Erzählen sie absichtlich oder unabsichtlich falsche Sachverhalte, dann können sie dafür zur Rechenschaft gezogen oder gar juristisch belangt werden.

Die literaturwissenschaftliche Erzähltheorie erklärt diesen kategorialen Unterschied von faktualen und fiktionalen Erzählungen, indem sie bei fiktionalen Erzählungen zwischen dem Autor als dem realen Sprecher dieser Erzählung und dem Erzähler als dem «fiktive[n] Aussagesubjekt der fiktionalen Rede» differenziert (Martínez \& Scheffel, 2012, S. 18). Diese "Grundunterscheidung" (Lahn \& Meister, 2008, S. 13) erklärt, warum journalistische Autoren, nicht aber literarische, für den Wahrheitsgehalt ihrer Erzählungen haften müssen. ${ }^{12}$ Günter Grass kann nicht für die Geschichten belangt werden, die Oskar Matzerath, der von ihm erfundene Erzähler der Blechtrommel, über sich und ande-

12 Da journalistische Beiträge nach dem Prinzip der Mehrfachautorenschaft produziert und publiziert werden (vgl. Bucher 2000), ist auch die Haftung für ihre Wahrheit dementsprechend modifiziert. Das Medienrecht legt fest, dass nicht nur die Autoren, sondern auch Redaktionen, Verlage und Sender für die Wahrheit und die Zulässigkeit journalistischer Beiträge haften müssen. 
re erzählt. ${ }^{13}$ Die Differenz von realem Autor und fiktivem Erzähler erklärt aber auch, warum für die Erzähler fiktionaler Texte die Innenwelten ihrer Figuren zugänglich sind, während sie für journalistische Erzähler - ungeachtet aller Experimente des literarischen Journalismus - letztlich unzugänglich bleiben (vgl. Klein \& Martínez, 2009, S. 2-5).

Andererseits kann gerade die Einbindung des Erzählers in die reale Welt erklären, warum es nicht nur bei fiktionalen, sondern auch bei faktualen Erzählungen Geschichten und Gegengeschichten geben kann, wie das die Plagiatsaffäre zeigt. Denn da die Erzähler faktualer Geschichten zur realen Welt gehören, sind sie auch in die Norm- und Wertesysteme ihrer Gesellschaften und Kulturen eingebunden, was wiederum ihre Sichtweise der Dinge bestimmt. Unterscheiden sich aber die Sichtweisen von Erzählern, dann unterscheiden sich auch ihre Geschichten. Das betrifft nicht nur die Darstellungsart, sondern auch die Geschichten selbst, sogar wenn diese auf den gleichen Sachverhalten beruhen.

Ein literaturwissenschaftliches Modell, das diesen Zusammenhang erfasst, ist das Sujet- bzw. Ereigniskonzept von Jurij M. Lotman. Nach Lotman hängt das Sujet, d.h. die erzählte Geschichte, «organisch (...) mit dem Weltbild [zusammen, knr], das den Massstab dafür liefert, was ein Ereignis ist» (Lotman, 1972, S. 333). Dieses Weltbild organisiert zunächst einmal die klassifikatorische semantische Ordnung, die die propositionale Struktur eines Textes bestimmt. Diese Ordnung ist anhand der räumlich-topographischen Zusammenhänge, die in einer Erzählung beschrieben werden, besonders gut $\mathrm{zu}$ erfassen, ${ }^{14}$ sie zeigt sich aber auch in allen anderen Klassifikationen. Ihr elementares Merkmal ist die Grenze. «Sie teilt den

13 Diese Differenzierung zwischen Autor und Erzähler ist allerdings kulturabhängig, wie das die Fatwa von Ayatollah Khomeini gegen Salman Rushdie und seinen Roman Die satanischen Verse beweist.

14 Das erklärt die grosse Bedeutung, die Lotman topographischen Strukturen zumisst (vgl. Renner 2004, 366).
Raum in zwei disjunkte Teilräume. Ihre wichtigste Eigenschaft ist ihre Unüberschreitbarkeit» (Lotman, 1972, S. 327). Ausgehend davon definiert Lotman den Begriff «Ereignis» als «die Versetzung einer Figur über die Grenze eines semantischen Feldes» (Lotman, 1972, S. 332; Hervorhebung beseitigt). ${ }^{15}$ Diese Figur ist dann der Handlungsträger, der «Held» der erzählten Geschichte (Lotman, 1972, S. 341). Da aber die Grenze vom Weltbild des Erzählers abhängig ist, sind auch das Ereignis und die erzählte Geschichte davon abhängig.

Lotmans paradoxe Ereignisdefinition als Überschreitung einer unüberschreitbaren Grenze erweist sich bei ihrer mengentheoretisch-logischen Rekonstruktion (vgl. Renner, 2004) als ein epistemologischer Sachverhalt, als ein Widerspruch zwischen dem, was ein Erzähler für wahr hält, und dem, was er beobachtet.

Die Grenze, die die klassifikatorische Ordnung einer Erzählung etabliert, lässt sich nämlich als eine Funktion von textspezifischen Ordnungssätzen darstellen. Syntaktisch gesehen sind das allquantifizierte Implikationen ${ }^{16}$, die mithilfe von Wenn-Dann-Beziehungen vorgeben, wel-

15 Lotmans Ereignisbegriff unterscheidet sich vom alltäglichen Ereignisbegriff in zweifacher Hinsicht. Er bezieht sich auf Ereignisse in einem emphatischen Sinn und nicht auf das, was eben so passiert. Auch ist er kein deskriptiver, sondern ein theoretischer Begriff, da er in ein theoretisches Konzept eingebunden ist. Ich versuche beidem dadurch Rechnung zu tragen, dass ich in meiner Terminologie zwischen Ereignis und Geschehen bzw. Geschehnis unterscheide.

16 Allquantifizierte Implikationen sind Sätze des Typs « $\wedge \mathrm{x}(\mathrm{Px} \rightarrow \mathrm{Rx})$ » (Für alle $\mathrm{x}$ gilt: Wenn $\mathrm{x}$ die Eigenschaft $\mathrm{P}$ hat, dann hat $\mathrm{x}$ auch die Eigenschaft R). In der Alltagssprache nehmen allquantifizierte Implikationen die Form von Allaussagen und Stereotypen an, z. B. "Alle Bayern trinken Bier». Die Beziehung zur klassifikatorischen Funktion von Lotmans Grenze ergibt sich dadurch, dass die allquantifizierte Implikation das prädikatenlogische Korrelat der Teilmengenrelation ist (die Menge $\mathrm{P}$ ist eine Teilmenge der Menge R) und Lotmans Grenze mengentheoretisch als nicht-triviale Teilmengenrelation interpretiert werden kann (vgl. Renner 2004, 363-366). 
che Figuren welchen Räumen bzw. Mengen angehören. Zugleich besitzen diese Ordnungssätze einen besonderen semantischen Status: Sie sollen im Rahmen der erzählten Geschichte immer wahr sein. In fiktionalen Erzählungen ist dieser Wahrheitsanspruch dadurch gegeben, dass der Autor bzw. Erzähler diese Geschichte so konstruiert hat. In faktualen Erzählungen beruht er darauf, dass diese Ordnungssätze vom Erzähler aufgrund seines Weltbildes, seiner Überzeugungen und Werte für wahr gehalten werden. Für die Figuren der erzählten Geschichte heisst das wiederum, sie sind an die Räume und Eigenschaften, die ihnen durch die Ordnungssätze zugewiesen wurden, gebunden. Daher können sie deren Grenzen nicht überschreiten. Aus diesem Grund muss das Geschehen - die Entwicklungen und die Wege der Figuren - mithilfe von Situationsbeschreibungen erfasst werden, die für jeden Zeitpunkt der Geschichte die Eigenschaften und Raumzugehörigkeiten der Figuren registrieren. Die Wahrheit dieser Situationsbeschreibungen beruht also auf Beobachtung und nicht auf Überzeugung. Sie ist korrespondenztheoretisch, nicht ideologisch begründet.

Ein Ereignis im Sinne Lotmans, das ergibt diese Rekonstruktion, liegt damit genau dann vor, wenn zwischen einem Ordnungssatz, dessen Wahrheit durch das Weltbild der Erzählers begründet ist, und einer Situationsbeschreibung, in der dieser Erzähler registriert, was tatsächlich der Fall ist, ein logischer Widerspruch besteht. Denn dann hat eine Figur eine Grenze überschritten, die nach den Spielregeln der erzählten Geschichte eigentlich unüberschreitbar ist. Versteht man eine $\mathrm{Ge}$ schichte wiederum als Abfolge von Ereignissen, zwischen denen chronologische und kausale Zusammenhänge bestehen, so lässt sich dies als ein Zusammenwirken von Situationsbeschreibungen und Ordnungssätzen darstellen. Die Situationsbeschreibungen erfassen das zeitliche Nacheinander und die Ordnungssätze legen die kausalen Zusammenhänge fest.

Der weitere Fortgang einer Geschichte kann dann unter Rückgriff auf das Gleichgewichtskonzept von Tzvetan Todorov (vgl. Todorov, 1972, S. 60) als Reparatur solcher Ordnungsverletzungen erklärt werden. ${ }^{17}$ Der Protagonist kehrt in seinen Raum zurück oder er verändert sich so, dass er in den neuen Raum passt. Eine dritte Möglichkeit ist, dass sich die semantische Ordnung durch eine Transformation so verändert, dass der Widerspruch verschwindet (vgl. Renner, 2004, S. 371-375). Ein weiteres Prinzip, das neben diesem Konsistenzprinzip den Fortgang einer Geschichte bestimmt, ist die Extrempunktregel. Sie besagt, dass durch die interne semantische Organisation der jeweiligen Teilräume ranghöchste Elemente ausgewiesen sind. Diese Extrempunkte bestimmen die Wege der Protagonisten innerhalb der Teilräume und markieren die Wendepunkte und Endpunkte von Geschichten (vgl. Renner, 2004, S. 375-377). ${ }^{18}$

Die Grenzüberschreitungstheorie bietet damit eine erzähltheoretische Erklärung, warum in journalistischen Erzählungen die gleichen Sachverhalte $\mathrm{zu}$ widersprüchlichen Geschichten verknüpft werden können, obwohl es sich nicht um fiktionale, sondern um faktuale Erzählungen handelt. Zugleich kann sie die Konstruktion von Geschichten aus Produzenten- wie aus Rezipientenperspektive erfassen, da sie auf ihrer elementaren Ebene mit mathematischen Mengen operiert und Mengen sowohl auf der Grundlage ausser- wie innertextlicher Gegebenheiten gebildet werden können.

Journalismustheoretisch folgt aus der Grenzüberschreitungstheorie, dass Journalisten die recherchierten Fakten nicht zu Geschichten verknüpfen können, ohne dabei auf Überzeugungen und Weltbilder zurückzugreifen. ${ }^{19}$ Journalistisches Erzählen kann daher schnell mit der Ob-

17 Dies entspricht dem Zusammenhang von Complication und Resolution bei Labov \& Waletzky 1967.

18 Zukunftsoffen sind Geschichten demnach, wenn sie zu einem Zeitpunkt erzählt werden, an denen Ordnungsverletzungen noch nicht behoben sind oder an denen ein Protagonist den Extrempunkt seines Weges noch nicht erreicht hat.

19 Ein vergleichbares Problem besteht bei der Auswertung statistischer Daten, etwa bei der Interpretation von Kurven. 
jektivitätsnorm - genauer gesagt mit dem Unparteilichkeitsprinzip des Journalismus - in Konflikt geraten, was wiederum seine Mehrfachsystemfähigkeit (vgl. Kohring, 2001, S. 79) tangiert. Dieses Konfliktpotenzial wird so lange nicht virulent, solange alle Mitglieder einer Gesellschaft ein gemeinsames Weltbild teilen und das berichtete Geschehen nach gleichen Kriterien beurteilen. Genau das aber ist bei Skandalen und in polarisierten Gesellschaften nicht der Fall.

\subsection{Geschichten und Gegengeschichten in der Plagiatsaffäre II}

Ausschlaggebend für die gegensätzlichen Standpunkte der beiden Lager in der Plagiatsaffäre ist, dass sie die Ungereimtheiten in Guttenbergs Dissertation nach divergierenden Kriterien klassifizieren. Das eine Lager beurteilt sie als Fehler in einer wissenschaftlichen Arbeit, das andere als Plagiat, als Diebstahl geistigen Eigentums und Betrug. Rekonstruiert man die beiden (Makro-)Geschichten, die sich daraus ergeben, dann liegen der Fehlergeschichte die beiden Ordnungssätze OS 1 und OS 2 zugrunde und der Betrugsgeschichte die Ordnungssätze OS 3 und OS 4. Dabei erfassen die Ordnungssätze OS 2 und OS 4 die unterschiedliche Bewertung dieser Ungereimtheiten, während die Ordnungssätze OS 1 und OS 3 die treibende Kraft für den Fortgang der jeweiligen Geschichte bilden.

, OS 1: Für alle x gilt:Wenn x den Doktortitel führt, dann setzt dies voraus, dass $\mathrm{x}$ eine Dissertation geschrieben hat, die keine gravierenden Fehler enthält.

, OS 2: Für alle $\mathrm{x}$ gilt: Übernimmt $\mathrm{x}$ in seine Dissertation Passagen aus anderen Arbeiten und kennzeichnet $\mathrm{x}$ diese Passagen nicht als Zitat, dann begeht $\mathrm{x}$ einen gravierenden Fehler.

, OS 3: Für alle x gilt: Wenn x Minister ist, dann darf $x$ nicht betrügen.

, OS 4: Für alle $\mathrm{x}$ gilt: Übernimmt $\mathrm{x}$ in seine Dissertation Passagen aus anderen Arbeiten und kennzeichnet $\mathrm{x}$ diese Passagen nicht als Zitat, dann begeht $\mathrm{x}$ einen Betrug.
Beide Geschichten beginnen damit, dass die Süddeutsche Zeitung am 16. Februar 2011 auf ihrer Titelseite einen Sachverhalt meldet, der nach OS 2 als Fehler und nach OS 4 als Betrug einzustufen ist und der damit entweder nur OS 1 oder auch noch OS 3 verletzt: "Nach Informationen der Süddeutschen Zeitung gibt es in Guttenbergs Dissertation einige Passagen, die wörtlich mit Formulierungen anderer Autoren übereinstimmen, ohne dass er dies wie vorgeschrieben gekennzeichnet hat» (SZ 16.2.2011). Diese Meldung, die als Situationsbeschreibung zu betrachten ist, ist aus gutem Grund so umständlich formuliert. Jede einfachere Formulierung brächte die Gefahr mit sich, einseitig Partei zu ergreifen. Die Fehlergeschichte endet dann am 21. Februar 2011 mit der Rückgabe des Doktortitels in Kelkheim, was die Verletzung von Ordnungssatz OS 1 behebt. Die Betrugsgeschichte endet mit dem Rücktritt Guttenbergs am 1. März 2011, womit die Verletzung von OS 3 beseitigt ist.

Methodisch lassen sich Ordnungssätze aus Textaussagen ableiten, die mehr oder minder deutlich als allgemein gültige Feststellungen und Regeln formuliert sind. ${ }^{20}$ Etwa wenn Guttenberg in seiner Rede sagt: «Ich bin ein Mensch mit Fehlern und Schwächen» (FAZ 22.2.2011) oder wenn der CSU-Vorsitzende Horst Seehofer feststellt: «Ein Minister stürzt nur, wenn es die eigene Partei will» (SZ 22.2.2011). Zum anderen sind Ordnungssätze auch indirekt fassbar, wenn Regelverletzungen formuliert werden oder wenn Sachverhalte eine besondere Aufmerksamkeit erregen. Beispiele sind die Formulierung Guttenbergs, er habe «gravierende Fehler» gemacht, die dem «wissenschaftlichen Kodex» nicht entsprechen (FAZ 22.2.2011), und der jubelnde Applaus, den beide Reportagen in Kelkheim verzeichnen. Die Situationsbeschreibungen wiederum lassen sich relativ einfach aus Berichten und Meldungen

20 Zur methodischen Anwendung von Lotmans Sujetkonzept und Grenzüberschreitungstheorie vgl. Krah 2006, 285-349; Renner 2012, 55-65; Titzmann 2013; Müller \& Grimm 2016. 
ableiten, da diese die Dinge auf ähnliche Weise registrieren.

Ein Vergleich aller drei Guttenberg-Geschichten ergibt, dass die Hetzjagdgeschichte einen anderen Weg einschlägt, um die Sachverhalte zu einer abweichenden Geschichte zu verbinden. Hier werden nicht identische Sachverhalte divergierend klassifiziert, sondern hier wird der zeitliche Rahmen so gewählt, dass sich dadurch eine andere Geschichte ergibt. $^{21}$

Die Hetzjagdgeschichte wird von den Guttenberg-Anhängern erzählt, um zu erklären, warum die Guttenberg-Gegner die Ungereimtheiten der Dissertation nicht als Fehler, sondern als Betrug einstufen. In den Medien wird sie von der BILD-Zeitung favorisiert (vgl. Renner, 2013, 280-283). Aber auch Berthold Kohler spielt in seinem Kommentar auf diese Geschichte an, wenn er schreibt: «[J]eden, der einen eigenen Kopf habe und aus dem Einheitsbrei der politischen Korrektheit herausrage, wollten Parteien und Medien kaputtmachen, schreit die gequälte Volksseele» (FAZ 23.2.2011). Unter Berücksichtigung der Artikel in der BILD-Zeitung lässt sich damit als Basis der Hetzjagdgeschichte Ordnungssatz OS 5 formulieren:

, OS 5: Für alle x gilt: Wenn x zum Berliner Politikbetrieb gehört, dann ist $\mathrm{x}$ keine herausragende Persönlichkeit, sondern gehört zum Einheitsbrei.

Dieser Ordnungssatz OS 5 wird nicht durch die Entdeckung der Plagiate, sondern durch den kometenhaften Aufstieg Guttenbergs verletzt. Das liegt zum Zeitpunkt der Plagiatsaffäre bereits zwei Jahre zurück. Guttenbergs Blitzkarriere begann 2009, als er im Alter von 38 Jahren zum Wirtschaftsminister berufen wurde. Darum werden jetzt nach der Logik der Hetzjagdgeschichte die Fehler in der Dissertation dazu benutzt, um ihn wieder klein zu machen.

Guttenberg beruft sich in der Kelkheimer Rede ebenfalls auf die Hetzjagdgeschichte und er transformiert sie in ein

21 Vgl. dazu die Ausführungen von Hans Krah zur «Grenzsetzung» (Krah 2006, 327-330).
Bewältigungsnarrativ (vgl. Lucius-Hoene \& Scheidt, 2017). Seine Metapher von der Wettertanne, die den Stürmen trotzt, operiert mit der gleichen vertikalen semantischen Struktur wie Ordnungssatz OS 5. Als "Wettertannen», so schreibt die FAZ vom 23.2.2011 in einem eigenen Erklärkasten, werden «allein stehende $\mathrm{Na}$ delbäume bezeichnet, die in exponierten Hochlagen Wind und Wetter trotzen» (frs. FAZ 23.2.2011). Guttenberg erklärt damit die Plagiatsaffäre zu einem Extrempunkt seiner Karriere und behauptet zugleich, dass sie nicht den Endpunkt seiner Karriere bildet, sondern einen Wendepunkt, von dem aus es weiter aufwärts geht, sobald der Sturm vorbei ist.

Dass die Plagiatsaffäre $\mathrm{zu}$ diesem Zeitpunkt nicht als Endpunkt von Guttenbergs Karriere erscheint, ist auch der Äusserung der Kanzlerin zuzuschreiben, sie habe ihn als Minister und nicht als wissenschaftlichen Assistenten berufen. «Sie achte darauf, ob er seine Arbeit als Minister erfüllen könne. «Und da sage ich ja»" (FAZ 22.2.2011). Damit versucht sie die Fehlergeschichte zum verbindlichen Masternarrativ der konkurrierenden Skandalgeschichten zu erklären und die Betrugsgeschichte durch eine Ordnungstransformation ausser Kraft zu setzen. ${ }^{22}$ Das gelingt aber nicht, weshalb in Kelkheim die Fehlergeschichte, jedoch nicht die Betrugsgeschichte ihr Ende gefunden hat. $^{23}$

22 Zur Ordnungstransformation durch eine zentrale Figur vgl. Renner 2004, 379, und Renner 2012, 73-79.

23 Eine wesentliche Rolle für die nicht nachlassende Überzeugungskraft der Betrugsgeschichte spielt die Online-Plattform Guttenplag, wo sich jedefrau und jedermann selbst ein Urteil darüber bilden können, ob die Dissertation als fehlerhaft oder als versuchter Betrug zu bewerten ist. 


\section{Das "Wie" des journalistischen Erzählens}

\subsection{Journalistisches Erzählen und journalistische Textsorten}

Das «Wie der Erzählung», so definieren Lahn und Meister den discours einer Erzählung, «ist die sprachliche Mitteilung, die uns der Erzähler von diesen Gegenständen und Ereignissen [dem «Was des Erzählung», knr] liefert» (Lahn \& Meister, 2008, S. 14). Während also das «Was der Erzählung» auf der semantischen Achse «Erzähltext-Geschichte-Welt» zu verorten ist, ist das «Wie» auf die pragmatische Achse «Erzähler-Erzähltext-Rezipienten» bezogen. Daher ist es über die Anforderungen der Kommunikationsgattung Journalismus hinaus noch von den spezifischen pragmatischen Funktionen der jeweiligen journalistischen Text sorten geprägt.

Exemplarisch für das journalistische Erzählen im engeren Sinne ist die Reportage. Ihre perlokutionäre Funktion ist es, «die Zuhörer/Leser am Geschehen geistig und emotional teilhaben, sie miterleben lassen durch die authentische Erzählung» (Haller, 1995, S. 62). Eine solche Vermittlung von «Erfahrungshaftigkeit» (experientiality) ist, wie Monika Fludernik unter Rückgriff auf die soziolinguistischen Untersuchungen von William Labov und Joshua Waletzky schreibt, eine wesentliche Funktion des alltäglichen Erzählens und eine zentrale Wirkungsabsicht vieler literarischer Texte. "Handlung, Intentionen und Gefühle sind alle Teil der menschlichen Erfahrung, die in Erzählungen berichtet und gleichzeitig evaluiert wird" (Fludernik, 2008, S. 122). Daher ist es nur folgerichtig, wenn die Autoren journalistischer Reportagen bei ihrem Storytelling weitgehend die gleichen Erzähltechniken verwenden wie die Autoren litera rischer Texte.

Die perlokutionäre Funktion von Nachrichten und Berichten besteht dagegen darin, die Rezipienten über das aktuelle Geschehen auf dem Laufenden zu halten. Deswegen bestimmt die Aktualität bei diesen journalistischen Textsorten nicht nur die Auswahl der Themen, son- dern auch die Gestaltung des discours. Nachrichten und Berichte sind nach dem Leadprinzip aufgebaut, sie informieren über das Wichtigste zuerst. Das erlaubt ein zeitsparendes Lesen und eine flüchtige Rezeption (vgl. Pöttker, 2003, S. 425). Anders als die Reportage dient diese Form des journalistischen Erzählens nicht der Vermittlung individueller Erfahrungen, sondern der nüchternen Darstellung der Fakten.

Die Ausdifferenzierung der verschiedenen kommunikativen Leistungen des Erzählens in den unterschiedlichen journalistischen Textsorten macht es weiterhin erforderlich, neben den informationsbetonten Textsorten auch die meinungsbetonten zu berücksichtigen. Denn die Evaluation des erzählten Geschehens durch den Erzähler ist, wie Labov und Waletzky zeigen, ein Grundbestandteil des alltäglichen Erzählens (vgl. Labov \& Waletzky, 1967, S. 33-39), ebenso ist sie ein fester Bestandteil des literarischen Erzählens (vgl. Lahn \& Meister, 2008, S. 104). In (informations-)journalistischen Erzählungen kann der Erzähler das dargestellte Geschehen jedoch nicht bewerten, das würde dem Prinzip der Trennung von Information und Meinung widersprechen. Hier werden die darstellende und die bewertende Funktion des Erzählens voneinander getrennt. Nachrichten und Berichte dienen der Darstellung des berichteten Geschehens, Kommentare, Glossen und Karikaturen seiner Evaluation. Damit ist auf der Ebene der einzelnen Texte die Trennung von Information und Meinung gewahrt und die evaluative Funktion des Erzählens wird auf die Ebene des journalistischen Diskurses verlagert, wo informationsbetonte und meinungsbetonte Beiträge miteinander interagieren.

\subsection{Die Gestaltung zeitlicher Zusammenhänge}

Journalistische Erzählungen im engen und im weiten Sinne unterscheiden sich bei ihrer erzählerischen Gestaltung deutlich voneinander, so bei der Wahl von Erzählperspektiven oder bei der Anlage der Figuren (vgl. Renner \& Schupp, 2017, S. 126127). Hier soll lediglich die Gestaltung der 
zeitlichen Zusammenhänge besprochen werden, da bei ihnen der Einfluss des Aktualitätsprinzips auf das journalistische Erzählen besonders deutlich wird. Inhaltlich lässt sich die Zeitgestaltung anhand der Beziehungen von erzählter Zeit und Erzählzeit erfassen (vgl. Martínez \& Scheffel, 2012, S. 32-49; Lahn \& Meister, 2008, S. 136-151), formale Aspekte der Zeitgestaltung sind der Gebrauch der grammatikalischen Zeiten und die Datumsangaben (vgl. Lahn \& Meister, 2008, S. 151-154).

Bei den Datumsangaben der Beispielstexte fällt auf, dass sie alle - Berichte, Reportagen und Kommentare - ausschliesslich relationale Zeitangaben verwenden, besonders häufig ist dabei der Bezug auf die Wochentage. Kalendarische Zeitangaben finden sich nur in den Kopfzeilen der Zeitungsseiten und manchmal in der Autorenzeile. So weist der Bericht in der Frankfurter Allgemeinen Zeitung vom 22. Februar 2011 in der Autorenzeile mit den Angaben «oge./ban./kau. BERLIN / KELKHEIM, 21. Februar» (die Jahreszahl fehlt) die Verfasser sowie Ort und Zeitpunkt des berichteten Geschehens aus. Im Fliesstext ist dann zu lesen, was Guttenberg "am Montagabend» gesagt hat und was die Universität Bayreuth «am Montagabend» mitgeteilt hat. Die Süddeutsche Zeitung vom 22. Februar 2011 berichtet über die Veranstaltung «am Montagabend» und erwähnt, dass «bereits an diesem Dienstag» in Bayreuth eine Kommission zusammentreten will. Der Referenzpunkt all dieser relationalen Zeitangaben ist also Dienstag, der 22. Februar 2011, der Publikationstermin der Zeitungsausgaben. Dieser Termin markiert die Hier-Jetzt-Ich-Origo (vgl. Bühler, [1934] 1999, S. 102) der journalistischen Sprecherinstanz und bildet den gemeinsamen Bezugspunkt von Erzähler und Leser, um die mitgeteilten Geschehnisse zeitlich zu verorten.

Als grammatikalische Zeit benutzen Erzählungen üblicherweise das Präteritum, da das Erzählte bereits vergangen ist (vgl. Lahn \& Meister, 2008, S. 151). Hiervon weichen die Kelkheim-Reportagen deutlich ab, bei ihnen dominiert das Präsens. Das ist auf die Wirkungsabsicht von Reportagen zurückzuführen, auf das Er- möglichen eines Miterlebens. Ihr Präsens ist ein historisches Präsens, wie es auch in literarischen Texten zur Imagination des Geschehens und zur Intensivierung des Erlebens verwendet wird (vgl. Lahn \& Meister, 2008, S. 153).

Die Berichte sind dagegen im Präteritum gehalten, mit einer bemerkenswerten Ausnahme: Ihr erster Satz steht im Perfekt oder im Präsens. Exemplarisch ist der Leadsatz im Bericht der FAZ vom 22. Februar 2011: «Bundesverteidigungsminister zu Guttenberg (CSU) hat schwerwiegende Fehler bei der Verfassung seiner Dissertation eingestanden, will aber als Verteidigungsminister im Amt bleiben.» Diese Besonderheit beruht auf einer konventionellen Norm des Zeitungsjournalismus, die Volker Wolff in seinem praktischen Handbuch folgendermassen wiedergibt: «Gilt das Berichtete noch (...), steht der erste Satz im Präsens. (...) Ist das Geschehen abgeschlossen, sind die Taten vollbracht, ist der Schaden eingetreten, das Wort gesprochen und hat das alles noch einen Gegenwartsbezug, dann steht der erste Satz im Perfekt»(Wolff, 2011, S. 60). Erzähltheoretisch gesehen bedeutet das, dass der Leadsatz die Funktion einer Situationsbeschreibung erfüllt, die den aktuellen Zustand des erzählten Geschehens für den Zeitpunkt registriert, an dem es erzählt wird.

Dieser formale Aspekt der Zeitgestaltung von Nachrichten und Berichten korrespondiert mit ihrer inhaltlichen Gestaltung. Da sie nach dem Leadprinzip organisiert sind, ist bei ihnen die natürliche Abfolge der erzählten Geschehnisse, die ordo naturalis, in eine textsortenspezifische ordo artificialis (vgl. Lahn \& Meister 2008, S. 138) transformiert. Die Reihenfolge, in der Nachrichten und Berichte das Geschehen erzählen, entspricht nicht dessen zeitlichem Nacheinander, sondern der Relevanz. Dabei sind Nachrichten nach dem Drei-Satz-Schema und Berichte nach dem Fünf-Absatz-Schema aufgebaut. Sie fassen ihre Informationen $\mathrm{zu}$ drei bzw. fünf Paketen zusammen, die zunächst die aktuelle Lage, dann die Details inklusive der zurückliegenden Geschehnisse und 
schliesslich zukünftige Sachverhalte behandeln (vgl. Wolff, 2011, S. 54 und 70).

Auch die Berichte über Kelkheim entsprechen diesem Schema. Titelzeilen und Leadsätze skizzieren die aktuelle Lage: «Guttenberg gibt 'gravierende Fehler) zu», ist die Überschrift in der FAZ vom 22.2.2011, «Guttenberg gibt seinen Doktortitel zurück» schreibt die SZ. Danach berichten sie im Einzelnen, was sich in Kelkheim zugetragen hat und welche Politiker sich jetzt zur Plagiatsaffäre geäussert haben. Nähere Informationen zum zurückliegenden Skandalgeschehen gibt es nicht, das ist den Lesern bereits bekannt. Im Zukunftsabsatz schreibt dann die FAZ, die SPD habe angekündigt, «es werde eine Aktuelle Stunde im Bundestag zu der Affäre geben", und die SZ berichtet über den aktuellen Stand von Guttenplag, wonach «mindestens ein Fünftel der Dissertation aus anderen Werken übernommen» wurde und diese Zahl «weiter steigen» dürfte, da die neuesten Funde «hier noch nicht berücksichtigt» seien.

Ruft man sich in Erinnerung, dass Nachrichten und Berichte aufgrund des Aktualitätsanspruchs des Journalismus dazu dienen, zukunftsoffene Makrogeschichten zu erzählen, so erscheint das Leadprinzip von Nachrichtentexten mitsamt seinen Tempus-Vorgaben für den ersten Satz wie eine Kollision der erzählten Zeit mit dem Zeitpunkt des Erzählens. Das Erzählen zukunftsoffener Geschichten ist kein übliches Erzählen, bei dem der Erzähler aus der Distanz auf ein abgeschlossenes Geschehen zurückblickt. Es ist ein gleichzeitiges Erzählen, das mit der aktuellen Situation des Erzählers unmittelbar verkoppelt ist. Dabei verschwimmen «die Grenzen zwischen handelndem Helden und schreibendem Erzähler, zwischen erlebendem und erzählendem Ich» (Martínez \& Scheffel, 2012, S. 77). Diese Entgrenzung ist der Ansatzpunkt der Pressionen, denen Journalisten immer wieder ausgesetzt sind. Und sie ist die Ursache eines dem aktuellen Informationsjournalismus immanenten Dilemmas: Da die Funktion des Journalismus die Selbstbeobachtung der Gesellschaft ist (vgl. Meier, 2007, S. 28), können Journalisten nur als Beobachter aktiv werden und dürfen sich nicht als aktiv Handelnde in das Geschehen einmischen. Genau das steht beim gleichzeitigen Erzählen in Frage.

\subsection{Journalistisches Erzählen und journalistischer Diskurs}

In diesem Dilemma verspricht die Trennung von Information und Meinung den Anspruch des aktuellen Informationsjournalismus einzulösen, als Beobachter der Gesellschaft $\mathrm{zu}$ agieren. Betrachtet man die Trias von Nachricht/Bericht, Reportage und Kommentar, wie sie in den überregionalen Tageszeitungen üblich ist, so leisten Nachrichten, Berichte und Reportagen die Beobachtung der aktuellen Ereignisse. Die einen informieren über das Geschehen und die anderen ermöglichen es, dies mitzuerleben. Die Kommentare greifen dagegen aktiv in den weiteren Fortgang des Geschehens ein. Sie bewerten es und zielen damit auf eine Veränderung oder eine Bestätigung der Einstellungen ihrer Rezipienten (vgl. Lüger, 1995, S. 28) mit den entsprechenden Konsequenzen für deren weitere Handlungen. Die logische Voraussetzung, dass diese intertextuelle Konstruktion zukunftsoffener Geschichten gelingt, besteht darin, dass journalistische Texte übereinstimmend auf die reale Welt als ihre gemeinsame Storyworld bezogen sind. Die publizistische Voraussetzung, dass die diskursive Auseinandersetzung über die Realisation von Handlungsalternativen mit dem journalistischen Anspruch vereinbar ist, neutraler Beobachter der Gesellschaft zu sein, besteht wiederum darin, dass in diesem journalistischen Diskurs die Meinungsvielfalt der Kommentare das Meinungsspektrum der Gesellschaft repräsentiert.

Ein Vergleich der verschiedenen Beiträge zur Kelkheim-Episode zeigt die besondere Bedeutung, die dem Leadprinzip für die diskursive Konstruktion zukunftsoffener Geschichten zukommt. Denn die Sachlage, die in den Leadsätzen der Nachrichten und Berichte als aktueller Stand der Makrogeschichte ausgewiesen ist, bildet zu einem den konstruktiven Rahmen der Mikrogeschichten, die in den Reportagen erzählt werden, und zum an- 
deren ist sie die argumentative Grundlage für die Evaluation des Geschehens in den kommentierenden Beiträgen.

So beginnt die Reportage in der Süddeutschen Zeitung mit einer Schilderung, wie Guttenberg am Tag nach Kelkheim von seinen Fraktionskollegen im Bundestag empfangen wird: "Sie wollen seine Hand schütteln, wollen ihn persönlich begrüssen; und wenn man sieht, wie viele das an diesem Nachmittag in Berlin wollen, dann sagt das schon einiges über die Frage, wie es dem Politiker Karl-Theodor zu Guttenberg geht» (Schmitz \& Braun, SZ 23.2.2011).

Die Reportage erzählt das (vermeintliche) Ende der Plagiatsaffäre als Geschichte einer Heimkehr. Genauer gesagt, sie stützt sich auf das Handlungsschema (vgl. Martínez \& Scheffel, 2012, S. 27) der Heimkehr des verlorenen Sohns. Guttenberg ist von seinem Irrweg heimgekehrt, er ist von seiner Position abgerückt, seine Dissertation besässe keine nennenswerten Fehler, und hat in Kelkheim öffentlich Busse geleistet: «Guttenberg kreuzigt sich jetzt selbst. Nimmt Schuld auf sich, wodurch ihm das Kunststück gelingt, an Achtung bei den Menschen zu gewinnen. Guttenberg bekennt demütig, Fehler gemacht zu haben» (Schmitz \& Braun, SZ 23.2.2011). Diese Geschichte kann man nur erzählen, wenn man glaubt, dass sich die Fehlergeschichte als die verbindliche Geschichte der Plagiatsaffäre durchgesetzt hat. Auch der Autor der Reportage in der Frankfurter Allgemeinen Zeitung ist davon überzeugt, dass Guttenberg nunmehr die Plagiatsaffäre überstanden hat, und schreibt als Überschrift: «Die fränkische Wettertanne nach dem Sturm» (Georgi, FAZ 23.2.2011). Aus heutiger Sicht weiss man, dass die richtige meteorologische Metapher die «Ruhe vor dem Sturm» gewesen wäre und dass es besser gewesen wäre, anstatt auf das Gleichnis aus der Bibel auf Thomas Manns Roman Die Bekenntnisse des Hochstaplers Felix Krull zurückzugreifen. ${ }^{24}$

24 Das wäre jedoch zum Zeitpunkt der Veröffentlichung dieser Reportage ein Verstoss gegen die Regeln der Verdachtsberichterstattung gewesen.
Der analysierende Beitrag von Günter Bannas in der Frankfurter Allgemeinen Zeitung vom 23. Februar 2011 zeigt exemplarisch, welche Bedeutung der Sachverhalt, den die Berichte in ihren Leadsätzen formulieren, für den Argumentationsaufbau kommentierender Beiträge, ja für den gesellschaftlichen Diskurs insgesamt besitzt. Sein Artikel beginnt folgendermassen: «Die Bundeskanzlerin findet die Entscheidung Karl-Theodor zu Guttenbergs, auf den Doktortitel zu verzichten richtig, hat Regierungssprecher Seibert am Dienstag offiziell die Haltung Angela Merkels beschrieben» (Bannas, FAZ 23.2.2011). Diese Sachverhaltsfeststellung ist auch die Prämisse des Kommentars von Berthold Kohler, der die nunmehr entstandene Situation negativ bewertet, da sie den Grundsätzen seines Weltbildes widerspricht:

\begin{abstract}
Noch aber ist Deutschland eine Republik, und noch ist ein Plagiat Diebstahl geistigen Eigentums. Die Kanzlerin mag aus naheliegenden Gründen über Letzteres hinweggehen, wenigstens nach aussen hin. Den Schaden im Kosmos der bürgerlichen Werte, den die Operation zur Rettung des gestrauchelten Bannerträges nach sich zieht, kann aber auch Frau Merkel unmöglich übersehen. Die Aktion, so sie denn gelingt und schon alles am Licht ist, wird Spätfolgen haben. (Kohler, FAZ 23.2.2011)
\end{abstract}

Wie am Beispiel der Kelkheim-Berichterstattung deutlich wird, stellt sich also das intertextuelle Zusammenspiel der journalistischen Textsorten, die auf der Ebene des journalistischen Diskurses die darstellende und die bewertende Funktion des Erzählens übernehmen, so dar, dass die (im engen wie im weiten Sinne) erzählenden Textsorten die informierende Funktion des Erzählens und die argumentativen Textsorten die evaluative ausführen. Dabei nehmen Nachrichten und Berichte eine vermittelnde Position ein. Denn die besondere kommunikative Potenz des Leadprinzips, Öffentlichkeit herzustellen (vgl. Pöttker, 2003, S. 425), besteht nicht nur darin, rasch über das Wichtigste zu informieren. Sie besteht auch darin, dass es die 
Darstellung des aktuellen Geschehens so komprimiert und abstrahiert, dass dieser Sachverhalt in der argumentativen Auseinandersetzung des gesellschaftlichen Diskurses als Fall klassifiziert und evaluiert werden kann. ${ }^{25}$ Das ist die spezifische Leistung des aktuellen Informationsjournalismus. Er organisiert das Erzählen zukunftsoffener Geschichten so, dass sich die gesellschaftliche Diskussion über den Fortgang dieser Geschichten auf beobachtbare Fakten stützen kann und nicht auf ein blindes Vertrauen in die Meinungsführer angewiesen ist.

\section{Schlussbemerkungen}

Es war das Ziel dieser Studie, am Beispiel der Berichterstattung über die Kelkheim-Episode aufzuzeigen, was die literaturwissenschaftliche Erzähltheorie zum Verständnis des journalistischen Erzählens beitragen kann. Ein wesentliches Ergebnis ist, dass sich diese Auseinandersetzung nicht auf das Storytelling, auf das Erzählen im engen Sinne, beschränken kann. Vielmehr erscheinen Storytelling und Leadprinzip als zwei komplementäre Erzählverfahren, die der Informationsjournalismus zur Darstellung und Konstruktion seiner zukunftsoffenen (Makro-) Geschichten verwendet. Dabei garantiert das intertextuelle Zusammenspiel der verschiedenen narrativen und argumentativen Textsorten, dass dieses journalistische Erzählen den Anforderungen des Journalismus entspricht.

Es bedarf keiner eigenen Begründung, dass die Ergebnisse dieser Fallstudie noch in weiteren Untersuchungen empirisch zu überprüfen sind. Auch müssen diese Untersuchungen über das journalistische Erzählen in überregionalen Tageszeitungen hinausgehen. Wie gestaltet sich das journalistische Erzählen in jenen Formen des Journalismus, die wie der Boulevardjournalismus oder der Sportjournalismus keine Trennung von Information und Mei-

25 Hier bestehen deutliche Übereinstimmungen mit dem Verhältnis von Erzählen und Argumentieren im juristischen Diskurs (vgl. von Arnauld 2009, 37; Hübner 2017). nung kennen? Welchen Einfluss haben die Medien auf das journalistische Erzählen? So kombiniert die charakteristische Textsorte des Zeitschriftenjournalismus, der Magazinbericht, narrative und argumentative Passagen miteinander (vgl. Wolff, 2011, S. 83-94), und die Moderatoren von Fernsehnachrichten erscheinen gerade$\mathrm{zu}$ als "Nachrichtenerzähler» (Hickethier, 1998, S. 187; Köhler, 2009). Dann ist aber auch zu fragen, inwieweit die Rezipienten journalistischer (Makro-)Erzählungen diese im Sinne der berichtenden Journalisten verstehen oder ob sie die einzelnen Bruchstücke des nach und nach erzählten Geschehens zu eigenen Geschichten zusammenfügen, die den von den Journalisten erzählten nicht entsprechen. ${ }^{26}$

Schon jetzt ist aber zu sagen, dass sich diese Fragen nicht mit punktuellen Erklärungen einzelner Phänomene beantworten lassen. Denn die Besonderheiten des journalistischen Erzählen sind, wie das diese Studie deutlich zeigt, das Ergebnis eines systemischen Zusammenhangs zwischen den kommunikativen Leistungen des Erzählens und den Anforderungen der Kommunikationsgattung Journalismus.

\section{Literatur}

Aumüller, M. (Hrsg.). (2012). Narrativität als Begriff. Analysen und Anwendungsbeispiele zwischen philologischer und anthropologischer Orientierung. Berlin: De Gruyter.

Bremond, C. (1972). Die Erzählnachricht. In J. Ihwe (Hrsg.), Literaturwissenschaft $u$. Linguistik. Bd. III (S. 177-217). Frankfurt am Main: Athenäum.

Bucher, H.-J. (2000). Journalismus als kommunikatives Handeln. Grundlagen einer handlungstheoretischen Journalismustheorie. In M. Löffelholz (Hrsg.), Theorien des Journalismus (S. 213-231). Wiesbaden: Westdeutscher Verlag.

Bühler, K. (1999). Sprachtheorie. Die Darstellungsfunktion der Sprache. Stuttgart: Lucius \& Lucius.

26 Ein aktuelles Beispiel ist die Fernsehberichterstattung über die Flüchtlinge im Herbst 2015 (vgl. Herrmann 2018). 
Burger, H. (1990). Sprache der Massenmedien. 2. Auflage. Berlin: De Gruyter.

Eberwein, T. (2013). Literarischer Journalismus. Theorie - Traditionen - Gegenwart. Köln: Von Halem.

Emery, M., \& Emery, E. (1992). The press and America. An interpretative history of the mass media. 7. Auflage. Englewood Cliffs: Prentice Hall.

Fludernik, M. (2008). Erzähltheorie. Eine Einführung. 2. Auflage. Darmstadt: WBG.

Fludernik, M., \& Ryan, M.-L. (Hrsg.) (2018). Narrative Factuality. A Handbook. Berlin: De Gruyter.

Früh, W., \& Frey, F. (2014). Narration und Storytelling. Theorie und empirische Befunde. Köln: Von Halem.

Greimas, A. J. (1972). Elemente einer narrativen Grammatik. In: Heinz Blumensath (Hrsg.), Strukturalismus in der Literaturwissenschaft (S. 47-67). Köln: Kiepenheuer \& Witsch.

Haller, M. (1995). Die Reportage. Ein Handbuch für Journalisten. 3. Auflage. Konstanz: UVK.

Herrmann, F. (2018). Das Verschwinden von Fakten aus der Berichterstattung. Überlegungen zur Analyse von Narrativen des öffentlichen Diskurses am Beispiel des Flüchtlingsthemas. In L. Blasch, D. Pfurtscheller \& T. Schröder (Hrsg.), Schneller, bunter, leichter? Kommunikationsstile im medialen Wandel (S. 37-52). Innsbruck: Innsbruck University Press.

Hickethier, K. (1998). Narrative Navigation durchs Weltgeschehen. Erzählstrukturen in Fernsehnachrichten. In K. Kamps \& M. Meckel (Hrsg.), Fernsehnachrichten. Prozesse, Strukturen, Funktionen (S. 185-202). Opladen: Westdeutscher Verlag.

Hübner, G. (2017). Überzeugen. In M. Martínez (Hrsg.), Erzählen. Ein interdisziplinäres Handbuch (S. 286-293). Stuttgart: J. B. Metzler.

Hühn, P., Pier, J., Schmid, W., \& Schönert, J. (Hrsg.) (2009): The living handbook of narratology. Abrufbar unter: http://www. lhn.uni-hamburg.de/ (letzter Aufruf: 8. April 2018).

Klein, C., \& Martínez, M. (2009). Wirklichkeitserzählungen. Felder, Formen und Funktionen nicht-literarischen Erzählens. In C. Klein \& M. Martínez (Hrsg.), Wirklichkeits- erzählungen. Felder, Formen und Funktionen nicht-literarischen Erzählens (S. 1-14). Stuttgart: J. B.Metzler.

Kohring, M. (2001). Vertrauen in Medien Vertrauen in Technologie. Arbeitsbericht. Stuttgart: Akademie für Technikfolgenabschätzung in Baden Württemberg.

Köhler, S. (2009). Die Nachrichtenerzähler. Zur Theorie und Praxis nachhaltiger Narrativität im TV-Journalismus. Baden-Baden: Nomos.

Krah, H. (2006). Einführung in die Literaturwissenschaft. Textanalyse. Kiel: Ludwig.

Kuhn, M. (2011). Filmnarratologie. Ein erzähltheoretisches Analysemodell. Berlin: De Gruyter.

Labov, W., \& Waletzky, J. (1967). Narrative analysis: Oral versions of personal experience. In J. Helm (Hrsg.), Essays on the verbal and visual arts. Proceedings of the 1966 Annual Spring Meeting of the American Ethnological Society (S. 12-44). Seattle: University of Washington Press.

Lahn, S., \& Meister, J.C. (2008). Einführung in die Erzähltextanalyse. Stuttgart: J. B. Metzler.

Lévi-Strauss, C. (1978). Die Struktur der Mythen. In C. Lévi-Strauss, Strukturale Anthropologie (S. 226-254). Frankfurt am Main: Suhrkamp.

Liesem, K. (2017). Der Verdacht. Drahtseilakt zwischen Medienfreiheit und Persönlichkeitsschutz. In K. N. Renner, T. Schultz \& J. Wilke (Hrsg.), Journalismus zwischen Autonomie und Mehrwert (S. 119-129). Köln: Von Halem.

Lotman, J. M. (1972). Die Struktur literarischer Texte. München: W. Fink.

Lucius-Hoene, G., \& Scheidt, C. E. (2017). Bewältigen von Erlebnissen. In M. Martínez (Hrsg.), Erzählen. Ein interdisziplinäres Handbuch (S. 235-242). Stuttgart: J. B. Metzler.

Lüger, H.-H. (1995). Pressesprache. 2. Auflage. Tübingen: Niemeyer.

Martínez, M. (Hrsg.) (2017). Erzählen. Ein interdisziplinäres Handbuch. Stuttgart: J. B. Metzler.

Martínez, M. (Hrsg.) (2011). Handbuch Erzählliteratur. Theorie, Analyse, Geschichte. Stuttgart: J. B.Metzler.

Martínez, M., \& Scheffel, M. (2012). Einführung in die Erzähltheorie. München: C. H.Beck. 
Meier, K. (2007). Journalistik. Konstanz: UVK.

Meier, O. (2004). Literatur und Journalismus. Ein Geschwisterstreit geht ins 21. Jahrhundert. Medienheft. Abrufbar unter: www. medienheft.ch/kritik/bibliothek/k22_MeierOliver.html. (Letzter Zugriff: 8. April 2018).

Müller, M., \& Grimm, P. (2016). Narrative Medienforschung. Einführung in Methodik und Anwendung. Konstanz: UVK.

Perrin, D. (2010). Wie sich Formate verändern: die Mustervariationen der Geschichte in Fernsehnachrichten. In H.-J. Bucher, T. Gloning \& K. Lehnen (Hrsg.), Neue Medien - neue Formate. Ausdifferenzierung und Konvergenz in der Medienkommunikation (S. 145-165). Frankfurt am Main: Campus.

Pöttker, H. (2003). Nachrichten und ihre kommunikative Qualität. Die «Umgekehrte Pyramide»- Ursprung und Durchsetzung eines journalistischen Standards. Publizistik, 48(4), S. 414-426.

Propp, V. (1975). Morphologie des Märchens. Frankfurt am Main: Suhrkamp.

Renner, K. N. (2013). Die öffentliche Konstruktion einer nicht-fiktionalen Geschichte. Eine Fallstudie am Beispiel der Plagiatsaffäre Guttenberg. In K. N. Renner, D. von Hoff \& M. Krings (Hrsg.), Medien - Erzählen - Gesellschaft (S. 265-300). Berlin: De Gruyter.

Renner, K. N. (2012). Rudimentäres Erzählen nicht-fiktionaler Ereignisse in fernsehjournalistischen Nachrichtenfilmen. Eine Fallstudie am Beispiel zur SPD-Führungskrise im März 2008. In M. Aumüller (Hrsg.), Narrativität als Begriff. Analysen und Anwendungsbeispiel zwischen philologischer und anthropologischer Orientierung (S. 75-134). Berlin: De Gruyter.

Renner, K. N. (2004). Grenze und Ereignis. Weiterführende Überlegungen zum Ereigniskonzept von J. M. Lotman. In G. Frank \&W. Lukas (Hrsg.), Norm-Grenze-Abweichung. Kultur-semiotische Studien zu Literatur, Medien und Wirtschaft. Festschrift für Michael Titzmann (S. 357-381). Passau: Stutz.

Renner, K. N. \& Schupp, K. (2017). Journalismus. In Martínez, M.(Hrsg.), Erzählen. Ein interdisziplinäres Handbuch (S. 122-132). Stuttgart: J.B.Metzler.

Robert, V. (2013). Serialisierte Erzählung und kulturspezifische Pressenarrative. Die «Wulff-Affäre» in der deutschen Presse.
DIEGESIS. Interdisziplinäres E-Journal für Erzählforschung, 2(2), S. 51-76. Abrufbar unter: https://www.diegesis.uni-wuppertal.de/index.php/diegesis/article/ view/138 (letzter Aufruf: 8. April 2018).

Ryan, M.-L. (2004). Introduction. In M.-L. Ryan (Hrsg.), Narrative across media. The languages of storytelling (S. 1-40). Lincoln: University of Nebraska press.

Ryan, M.-L. (2013). Transmediales Storytelling und Transmedialität. In K. N. Renner, D. von Hoff \& M. Krings (Hrsg.), Medien -Erzählen-Gesellschaft (S. 88-117). Berlin: De Gruyter.

Titzmann, M. (2013). Narrative Strukturen in semiotischen Äusserungen. In H. Krah \& M. Titzmann (Hrsg.), Medien und Kommunikation. Eine interdisziplinäre Einführung (S. 115-141). Passau: Stutz.

Thon, J.-N. (2016). Transmedial narratology and contemporary media culture. Lincoln: University of Nebraska press.

Todorov, T. (1972). Die Grammatik der Erzählung. In H. Gallas (Hrsg.), Strukturalismus als interpretatives Verfahren (S. 57-71). Darmstadt: Luchterhand.

von Arnauld, A. (2009). Was war, was ist - und was sein soll. Erzählen im juristischen Diskurs. In C. Klein \& M. Martínez (Hrsg.), Wirklichkeitserzählungen. Felder, Formen und Funktionen nicht-literarischen Erzählens (S. 14-50). Stuttgart: J. B.Metzler.

Wilke, J. (2016). Von der Wahrhaftigkeit zur Gerechtigkeit. Die historische Herausbildung von Normen des journalistischen Handelns. Jahrbuch für Kommunikationsgeschichte, 18, S. 24-50.

Wolff, V. (2011). Zeitungs- und Zeitschriftenjournalismus. 2. Auflage. Konstanz: UVK.

\section{Quellen}

Bannas, G. (2011, 23. Februar). Vertrauen ist gut - aber kein Blankoscheck. Frankfurter Allgemeine Zeitung, S. 6.

Georgi, O. (2011, 23. Februar). Die fränkische Wettertanne nach dem Sturm. Frankfurter Allgemeine Zeitung, S. 6.

Kohler, B. (2011, 23. Februar). Entlassen. Frankfurter Allgemeine Zeitung, S. 1.

N. N. (2011, 16. Februar). Plagiatsvorwurf gegen Guttenberg. Süddeutsche Zeitung, S. 1. 
N. N. (2011, 22. Februar). Guttenberg gibt «gravierende Fehler» zu. Frankfurter AllDer Atgemeine Zeitung, S .1.

N. N. (2011, 22. Februar). Guttenberg gibt seinen Doktortitel zurück. Süddeutsche Zeitung, S. 1. Frankfurter Allgemeine Zeitung. Mittwoch 23.2.2011

N. N. (2011, 23. Februar). Ein Baum von einem Minister: Guttenbergs Wettertanne. Frankfurter Allgemeine Zeitung, S. 6.

Schmitz, T. \& Braun, S. (2011, 23. Februar). Der Doktor und das liebe Volk. Süddeutsche Zeitung, S. 3.

Schultz, T. (2011, 23. Februar). Prüfungsdruck. Süddeutsche Zeitung, S. 3.

Wagner, F. J. (2011, 17. Februar). Lieber Dr. zu Guttenberg. BILD. Abgerufen auf http:// www.bild.de 